\section{H. INFLUENZAE MENINGITIS}

\author{
BY
}

\author{
H. W. EVERLEY JONES, M.B., M.R.C.P. \\ Registrar to the Children's Department, St. Thomas's Hospital
}

In view of the recent interest shown in this condition it seems fitting to record the following six cases which have been treated in St. Thomas's Hospital during the last fifteen years. The records of all departments of the hospital have been reviewed, but the only cases found were those in children, no adult cases being recorded.

\section{Case 1}

E. H., a female, aged 1 year 3 months, and a two months premature baby, had had bronchopneumonia at 10 months She was admitted on July 23, 1922, with one day's history of malaise, restlessness, and "stiffness." She had vomited once.

State.-Temperature $103.3^{\circ}$, pulse 144 , respirations 35 . Drowsy, but irritable when roused. The anterior fontanelle was tense. There was a moderate degree of neck rigidity and occasional convulsive movements of the right hand. No other physical signs were present, and the lungs were clear. The cerebrospinal fluid was turbid and under raised pressure. A very large number of cells were present, nearly all polymorphs. $H$. influenzae was seen in a direct smear and on culture. Blood culture showed $\boldsymbol{H}$. influenzae.

Progress.-The patient ran a high remittent temperature, and in spite of daily lumbar punctures died on August 8, after an illness of thirteen days.

Necropsy.-The vertex and base of the brain were covered with plaques of thick yellow exudate. There was some degree of internal hydrocephalus, and the brain was congested. The upper lobe of the right lung was collapsed, but there was no sign of pneumonia. The liver was large, pale, and fatty. Smears and cultures of the pus showed $H$. influenzae.

\section{Case 2}

J. S., male, aged 11 months, was admitted on January 7, 1925, with several days' history of vague lassitude and irritability. Temperature, pulse, and respirations were normal, and meningitis was not suspected until January 20 , when he had, in addition to the above, definite neck rigidity and his temperature began to rise $\left(101^{\circ}\right)$. A lumbar puncture revealed a cerebrospinal fluid which was under raised pressure and was slightly turbid. Microscopy showed pus cells ++ , and no organisms were seen in a direct smear. On culture $H$. influenzae was shown to be the causative organism. His blood serum agglutinated $H$. influenzae in a titre of 1 in 100 . He was lumbar-punctured six times during the next nine days, but became comatose and died on January 29 , his temperature rising to $106^{\circ}$ before death. The duration of the disease was about twenty-five days.

Necropsy.-There was a thick yellow exudate on the base of the brain and some purulent patches over the vertex. $H$. influenzae was demonstrated in the exudate. The other organs were normal except for a pale fatty liver.

\section{Case 3}

M. S., a female, aged 8 months, a perfectly normal child, fell off a chair on to her head on March 21, 1930. On being picked up she vomited immediately, and continued to do so nearly every half-hour. She was admitted to hospital on Mareh 22.

State.-Very drowsy; temperature $101^{\circ}$, pulse 142 . No other physical signs were present. On March 23 the pulse rate rose to 200 and the temperature became subnormal; she became comatose and died after a total illness of two days.

Necropsy.-There was a purulent meningitis, chiefly basal. Nothing else was found and there was no cranial fracture. $H$. influenzae was isolated from the exudate.

\section{Case 4}

W. N., a male, aged $2 \frac{1}{2}$ months, was a six weeks premature baby, breast-fed. For two weeks he had had a cough, which was becoming worse. During the last few days he had been restless, and for two days his stools had been green. There was no one in the house with influenza. He was admitted to hospital on June $15,1933$.

State.-Temperature $98.4^{\circ}$, pulse 175 , respirations 80 . Distressed; breathing shallow; faintly cyanosed. The chest showed diffuse coarse rales and rhonchi. He was treated in the open air and given stimulants and expectorants, but showed no improvement. On June 24 the temperature had risen to $104.5^{\circ} \mathrm{F}$., the pulse to 196 , and the respirations to 140 , and he was extremely distressed. The physical signs were unchanged. On June 27 generalized convulsions started, and these persisted until death on June 29 . No other physical signs developed. The duration of the illness was about twenty-eight days.

Necropsy.-There was an excess of turbid fluid at the base and over the vertex of the brain, which was soft. The lungs showed bronchitis. A smear of the cerebrospinal fluid showed $H$. influenzae. Culture produced $H$. influenzae, B. coli, and Staphylococcus albus.

\section{Case 5}

G. H., a female, aged 1 year, was quite normal until three weeks previously, when she had a sudden onset of pyrexia with shivering. The patient was admitted to a local hospital looking pale and ill. While there she ran a remittent temperature (up to $104^{\circ}$ ), but presented no physical signs. She was transferred to St. Thomas's on January 27, 1937.

State.-Temperature $101^{\circ}$, pulse 125 , respirations 42. Pale; lay in bed curled up on her side; drowsy, but irritable when disturbed; fontanelle flat; slight degree of neck rigidity; Brudzinski's sign +. There were no localizing signs in the central nervous system, and the other systems appeared normal. The cerebrospinal fluid was clear and under slightly raised pressure. On standing, a fine cobweb coagulum settled. The cells were increased and were mostly lymphocytes. The protein was 0.045 per cent., and the chlorides $66 \mathrm{mg}$. per $100 \mathrm{c.cm}$. No tubercle bacilli were found in the coagulum, and the fluid was cultured. A radiograph of the chest was negative. On the 29 th the cerebrospinal fluid was still clear, but there was no coagulum and there was a higher percentage of polymorph cells. On February 2 she became comatose, and without further physical signs died. $H$. influenzae was seen on the culture plate that day.

Necropsy.-Thick creamy pus, blue-green in colour, was present at the base of the brain, and to a less extent over the vertex. A small abscess, $1.5 \mathrm{~mm}$. in diameter, was found just behind the posterior horn of the left lateral ventricle. The brain was very soft. The pus contained polymorph cells ++ and bacilli identical with $H$. influenzae. The lungs showed bronchitis. The other systems were normal.

\section{Case 6}

P. M., a male, aged 2 years 10 months, was a perfectly healthy child who had spent all his life in the Cromwell Nursery (Mothercraft Training Society). In the past he had had a mild otitis at the age of $1 \frac{1}{2}$ years. His mother had. just recovered from an attack of influenza, contracted two weeks before. He was admitted on May 10, 1937, with one day's history of vomiting, screaming, and feverishness.

State-Temperature $101.4^{\circ}$, pulse 126 , respirations 58. Flushed; lay curled up on his side; drowsy, but irritable when disturbed. Apart from neck rigidity and slight photophobia there were no abnormalities in his central nervous system. The ears, throat, and lungs were normal, and the abdomen rather carinated. The spleen was not enlarged and there was no rash. The cerebrospinal fluid was under great pressure and was turbid. Polymorph cells were present in large numbers with some endothelial cells. No organisms were seen in a direct smear, and the fluid was plated out. 
$20 \mathrm{c.cm}$. of anti-meningococcal serum was given. On May 11 he was more drowsy and the neck rigidity more marked; Kernig's sign + . The cerebrospinal fluid was similar to that of the day before. Intrathecal anti-meningococcal serum (20 c.cm.) was repeated. On the 12 th he was less drowsy; the cerebrospinal fluid was under normal pressure and was clearer. A white cell count showed a total of 13,000 , with 85 per cent. of polymorphs. $H$. influenzae was grown from the first specimen of cerebrospinal fluid. On the 13th the cerebrospinal fluid was almost clear. On May 18 a serum rash developed, but the patient was now sitting up and talking. The neck rigidity had disappeared. A blood culture, after six days' incubation, was sterile. The serum rash soon cleared and he made an uneventful recovery. On discharge there were no physical signs and the cerebrospinal fluid was clear. (This child was given sulphonamide $P$, half a tablet twice daily, from the second day.)

\section{Aetiology}

Meningitis due to the $H$. influenzae is not such a rare condition as might be supposed. Thus Neal (1934) and Huntington and Wilkes-Weiss (1936) found that it ranked fourth as a cause of purulent meningitis. At St. Thomas's (from 1922 to 1937), in children up to 8 years of age, a similar incidence was found: meningococcal meningitis, thirty-seven ; pneumococcal meningitis, fourteen ; streptococcal meningitis, seven; $H$. influenzae meningitis, six. The age incidence is important; the majority of the patients being under 2 years, and the year with the highest incidence is $0-1$. In the above series only one patient was over 2 years old.

Most cases are thought to be "primary" - that is, not to be a complication of ordinary influenza. A point in favour of this is the fact that in years when influenza has assumed epidemic proportions there has not been an increase in the number of cases of $\boldsymbol{H}$. influenzae meningitis. Actually in 1918 only one case of this type was reported. In many cases, however, there appears to be a definite association with otitis or pneumonia. In Case 4 the chest condition was the prominent feature, and in ten out of forty-two infantile cases quoted by Huntington and Wilkes-Weiss (1936) the association seemed more than coincidence.

\section{Clinical Course}

The onset may be sudden or gradual, more often the former. In the cases reported above four may be said to have started suddenly. The condition may be acute or subacute, but no case of chronic meningitis due to $H$. influenzae has been recorded. The course varies from a minimum of twenty-four hours, in the case of an infant cited by Huntington and Wilkes-Wefss (1936), to two or three months. In a case recorded by Wood (1932), in which recovery took place, two months elapsed before the cerebrospinal fluid became sterile. The usual duration is about two to four weeks.

Clinically the condition does not differ from meningococcal meningitis, but most cases occur in infancy, and the signs are frequently suggestive of extrameningeal infection. Pyrexia, which is often remittent and combined with a rapid and irregular pulse (Case 5), diarrhoea and vomiting, drowsiness or irritability, may be the presenting signs. Usually the anterior fontanelle is found to be tense. The most constant signs appear to be irritability and neck rigidity. Local signs are uncommon, oculomotor paresis being most often present.

Gibbens (1931) describes two clinical types: (1) meningitis ; (2) meningitis complicated by arthritis, which appears to be much rarer. Moreover, he states that the arthritis may occur alone when no bone change takes place, whereas when the two are combined erosion of bone may be a feature.

\section{Pathological Investigations}

Blood.-In practically all cases a polymorph leucocytosis takes place. Huntington and Wilkes-Weiss (1936) cite six cases out of the forty-five in which a leucopenia was noted ; but only one count was recorded, and that was at the start of the illness, so probably a higher count would have followed.

Blood Culture.-A positive result is not infrequently obtained. In a combined series of forty-three attempts, nineteen were positive. In Case 1 the organism was grown, and failure resulted from the one attempt in Case 6. If the procedure was repeated in every case there would probably be a high incidence of positive results.

Cerebrospinal Fluid.-The fluid is usually under a raised pressure, and varies from faint haziness to frank pus. Although the cells are almost invariably polymorphs, on rare occasions there may be large numbers of lymphocytes (Case 5, in which the fluid was quite abnormal). The total protein is raised and the chlorides lowered, though not to the extent seen in tuberculous meningitis. Microscopically, the causative organism may be seen in a direct smear, but not infrequently, as in Cases 5 and 6 , no bacteria are to be found until the cerebrospinal fluid has been cultured. For culture a medium containing blood is necessary, and several days may elapse before a growth appears. It is possible that some of the cases of "meningitis cause unknown" may be due to the apparent absence of $\boldsymbol{H}$. influenzae in the direct smear. The $\boldsymbol{H}$. influenzae is said to produce indole, but as $B$. coli, admittedly a rare cause of meningitis, does this, indole production cannot be considered to be pathognomonic.

\section{Post-mortem Findings}

There is engorgement of the superficial vessels of the brain, and vascular thrombi are common. A thick purulent exudate collects round the base of the brain and to a lesser extent over the sides and vertex, and may even extend down the spinal cord. Degeneration of the superficial layers of the cerebrum is usual, and in many cases areas of necrosis and even small abscesses, as in Case 5, may be present. The ventricles are distended, and should the basal exudate organize a considerable degree of internal hydrocephalus may result. The other organs usually show nothing of note, except that fatty degeneration of liver and kidneys is almost invariable. The association with otitis and pneumonia has been mentioned.

\section{Complications}

Besides remissions and relapses, both of which may occur, the following have been described: bronchopneumonia, sinusitis, otitis media, suprarenal haemorrhage, arthritis (Gibbens, 1931), superficial cutaneous abscesses, cellulitis, pleural effusion, pericarditis and endocarditis, and peritonitis (Williams, 1934). All these complications appear to be rare.

\section{Prognosis}

This is bad, but is better in the comparatively infrequent cases occurring in older children and adults. Bloom (1931) collected 302 cases from the literature up to 1930, and found a total mortality of 92.05 per cent. In Rivers's (1922) series of 220 cases 92 per cent. proved fatal. Of 
these 71 were over 2 years of age, and 97 were under 2 . In all I can find only fifty cases of recovery reported, including Case 6 above. Only four of these were under 1 year old.

\section{Treatment}

Much controversy has centred round the subject of specific treatment. The convalescent serum taken from patients who have recovered from influenza is generally thought to be useless, as this disease is almost certainly caused by a filterable virus with $H$. influenzae as a secondary invader.

Throat strains of $\boldsymbol{H}$. influenzae form a heterogeneous group of antigens, whereas nearly all strains of meningeal $H$. influenzae have similar antigenic properties (Pittman, 1933). Pittman also showed that all meningeal strains possessed a similar type of soluble specific antigen derived from the supernatant fluid from a centrifuged culture. Serum prepared without the addition of this soluble specific substance has been demonstrated (Wilkes-Weiss, 1936) to have little bactericidal action. To obtain an efficient antiserum it is essential to use both a fresh strain of the organism and the soluble specific substance. Horses have been thus injected in increasing doses, and the serum so obtained used in treatment.

In this disease Ward and Fothergill (1932) have shown that complement is absent from the cerebrospinal fluid, and so the injection of normal human serum is advised. The doses recommended (Ward and Fothergill, 1932) are 6 to $8 \mathrm{c.cm}$. of normal human serum plus $15 \mathrm{c.cm}$. of antiserum twice daily-one injection into the lumbar theca and the other into the lateral ventricle. Should the blood culture be positive they recommend an injection of 50 c.cm. intravenously.

The results of this treatment are disappointing. Of fifty cases treated in this way (Ward and Wright, 1932; Pittman, 1933 ; Huntington and Wilkes-Weiss, 1936; Ward and Fothergill, 1932) only three recovered. This shows no improvement over ordinary methods. It was noticed, however, that in several of these patients there was temporary improvement which was not sustained.

From these results it appears that treatment on these lines will prove of no avail. Repeated lumbar puncture is, in our present state of knowledge, the only measure of practical use, even forced spinal drainage being no more successful. Sulphonamide $\mathbf{P}$ was given in Case 6. Probably the dosage was not sufficient to enable conclusions to be drawn from the result, but the fact that recovery did occur suggests that this drug should be given a further trial.

\section{Summary}

1. Six cases of meningitis due to $H$. influenzae are reported, with one recovery.

2. An account of the condition is appended.

Grateful acknowledgements are due to Dr. R. C. Jewesbury, in whose ward all the cases were treated, and to Dr. J. Bamforth, who performed the pathological investigations.

\section{REFERENCES}

Bloom, C. J. (1931). New Orleans med surg. J., 83, 455

Gibbens, J. (1931). Lancet, 1, 291.

Huntington, R. W., and Wilkes-Weiss, D. (1936). J. Paediat., 9 $449,456,462$.

Neal, J. B., et al. (1934). J. Amer. med. Ass., 102, 513.

Pittman, M. (1933). J. exp. Med., 58, 683.

Rivers, T. M. (1922). Amer. J. Dis. Child., 24, 102.

Ward, H. K., and Fothergill, L. D. (1932). Ibid., 43, 873. and Wright, J. (1932). J. exp. Med., 55, 223

Williams, J. W. (1934). Amer. J. Dis. Child. 48, 840.

Wood, P. H. (1932). British Medical Journal, 2, 101.

\section{Clinical Memoranda}

\section{Strangulated Hernia of the Ovary in a Child of 6 Weeks}

In the British Medical Journal of August 7 last, Dr. M. H. Fridjohn and $\mathrm{Mr}$. Maurice Lee report a case of strangulated hernia of the ovary in a girl of 3 months; and, finding that "a careful survey of the literature reveals no case of a similar nature in so young a patient," they issue a request for details regarding any comparable case. As the father of a child who suffered from strangulated hernia of the ovary at the age of exactly 6 weeks I am in a position to record a case occurring at an even earlier age than that referred to by Dr. Fridjohn and Mr. Lee. Since, however, the event in question occurred ten years ago, and since I must rely upon my memory (fortunately checked by that of my wife) in attempting to comply with the request for "details," I trust that due allowance will be made for any inadequacy in my account.

At birth the girl in question was a healthy baby of five and a half pounds and showed no signs whatsoever of physical abnormality. Her progress was satisfactory until shortly before the development of the hernia, when feeding difficulties began to cause some concern. It may be confidently stated, however, that these difficulties were quite independent of the hernia which developed; and no vomiting occurred. At about 10 a.m. on the day on which the child became 6 weeks old the nurse in attendance reported the presence in the left groin of a lump, which had not been present when the child was last "changed." As in the case reported by Dr. Fridjohn and Mr. Lee, this lump was about as large as a walnut. After the lapse of ten years $I$ find it impossible to make any definite statement regarding the consistency of the swelling; but my impression is that it was moderately hard. It may be definitely stated, however, that the swelling was situated in the region of the left inguinal canal, that when first seen it proved to be irreducible, and that the child cried when it was palpated. Dr. Douglas Miller, who had attended the confinement, was summoned in the first instance; and the child was removed to a nursing home, where Mr. Walter Mercer operated about 2 p.m. The swelling was then found to have been due to a strangulated inguinal hernia of the left ovary, to which a developmental cyst was attached. The cyst was removed; the ovary, which was found to be healthy, was returned to the abdominal cavity; and the canal was closed.

The child made a good recovery, although some time elapsed before the feeding difficulties already mentioned were satisfactorily overcome. Her subsequent health has been excellent; and the only other serious illness from which she has suffered was an attack of acute appendicitis, necessitating appendicectomy, at the age of 8 .

When the case described is compared with that recorded by Dr. Fridjohn and $\mathrm{Mr}$. Lee the following features attract attention: (1) The hernia would appear to have occurred within a few hours of its discovery; (2) a developmental cyst was attached to the ovary; (3) the presence of the cyst and the patency of the inguinal canal, which enabled the hernia to occur, may be presumed to have been causally related, if not actually elements in the same developmental anomaly; (4) in spite of the presence of the cyst the affected ovary itself was of normal appearance and consequently did not require to be removed; (5) vomiting played no part in the clinical picture.

Edinburgh.

W. R. D. FAirbairn, M.D. 